\title{
Reduciendo desigualdades. Otros derechos y otros desarrollos
}

\author{
Reducing Inequalities: Other Rights and Other Developments
}

\author{
Ferrer, N. (editora académica). Cartagena de Indias (Bolívar): \\ Universidad San Buenaventura Cartagena, 2020, 178 p.
}

Germán Andrés Molina Garrido, ${ }^{1}$ Ph.D (c)

Copyright: ๑ 2021

Revista Internacional de Cooperación y Desarrollo. Esta revista proporciona acceso abierto a todos sus contenidos bajo los términos de la licencia creative commons Atribución-NoComercial-SinDerivar 4.0 Internacional (CC BY-NC-ND 4.0)

Tipo de artículo: Reseña

Recibido: enero de 2021

Revisado: febrero de 2021

Aceptado: marzo de 2021

\section{Autor}

1 Politólogo de la Universidad Nacional de Colombia; Magíster en Antropología de la Universidad de los Andes (Colombia) y Candidato a Doctor en Humanidades. Humanismo y Persona de la Universidad de San Buenaventura. Docente e investigador del Departamento de Humanidades de la Universidad El Bosque, miembro fundador de Surcos: Red internacional de territorios y territorialidades en América Latina y miembro del Crupo de Investigación Violencias, procesos educativos y éticas de la coexistencia de la Universidad Nacional de Colombia.

Correo electrónico: molinagerman@unbosque.edu.co

\section{Cómo citar:}

Molina Garrido, G. (2021). Reduciendo desigualdades. Otros derechos y otros desarrollos [reseña del libro Reduciendo desigualdades. Otros derechos y otros desarrollos de F. Nina (ed.)]. Revista Internacional de Cooperación y Desarrollo. 8(1), 135-138

DOI: $10.21500 / 23825014.5363$

\section{G OPEN ACCESS}

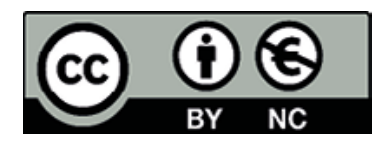

\section{Resumen}

Este documento es una reseña acerca del libro Reduciendo desigualdades. Otros derechos y otros desarrollos, bajo el liderazgo editorial de Nina Ferrer Araujo, decana de la Facultad de Derecho de la Universidad de San Buenaventura, en Cartagena de Indias (Colombia). La publicación emerge en un panorama intelectual caracterizado por la urgencia que tiene nuestra época de reformular vocabularios, conceptos, metáforas y paradigmas científicos en un momento de la historia colombiana, latinoamericana y mundial en el que la Academia crítica -por consenso explícito o implícito- parece querer decir que, ante el imperio del Capital, este mundo no da para más. Como tal, la reseña se ocupa de responder a las siguientes tres preguntas: 1. ¿Cuál es la transición científica de la que se ocupa este libro? 2. ¿Cuál es el orden de exposición que nos proponen sus páginas? y 3. ¿Cuál es la visión de fondo que nutre a la obra en general y que por ende se refleja en cada capítulo? El documento concluye que esta publicación es altamente recomendable, necesaria, deseable y que, vista con ojos de esperanza, podría estar abriendo camino hacia una nueva concepción del derecho, más próxima a las realidades del Caribe colombiano y, por tanto, a las geografías físicas y simbólicas de nuestros sures.

Palabras clave: desigualdad económica; desigualdad racial; derecho al desarrollo; derechos socioeconómicos; violencia económica; justicia.

\section{Abstract}

This paper is a book review of "Reducing Inequalities. Other Rights and Other Developments," under the editorial leadership of Nina Ferrer Araujo, Dean of the Faculty of Law at Saint Bonaventure University, Cartagena de Indias, campus, Colombia. The publication emerges in an intellectual panorama, which is characterized by the urgency of our time in order to reformulate scientific vocabularies, concepts, metaphors, and paradigms, at a time, in which the Colombian, Latin American, and World history, in which the critical Academy -by explicit or implicit consensus-seems to mean that, before the empire of Capital, 
this world is to not avail. As such, this book review is concerned with answering the following three questions: 1 . What is the scientific transition, which is the subject of this book? 2. What is the order of exposure, which this book proposes us? and 3. What is the underlying vision, which nourishes the work, in general, and is therefore, reflected in each chapter? The document concludes with the idea that this publication is highly recommended,

El momento transicional por el que hoy atraviesan las denominadas ciencias naturales, humanas, sociales y jurídico-políticas no es, ni mucho menos, una cuestión de interés reciente que acaso pudiera leerse como una respuesta, o como una mera reacción de rearticulación, frente a los sucesos cada vez más desafiantes que han acaecido en los tiempos de la pandemia global por la Covid-19. Por el contrario, pandemias mucho más antiguas tales como el racismo, el sexismo, el clasismo, el prejuicio especista que prioriza y superpone los intereses humanos frente a la cuestión animal o ambiental y, sobre todo, las violencias de género, por citar solo algunas de las más protuberantes de las que aún perviven y coexisten en los tiempos que corren, y cuyas genealogías nos remiten en algunos casos a la historia de la Conquista y la Colonización del "resto del mundo" por parte de Europa, y en otros a la matriz misma de la historia humana estudiada por la antropología como disciplina, están en la base de un amplio movimiento de reformulación de las ciencias y de las disciplinas científicas.

En un sentido amplio, se trata entonces de una transición científica cuya noticia parece haber sido anunciada con fuerza, rigor y claridad por lo menos a partir de la década del setenta del sigloxx. Esto es lo que algunos, como por ejemplo el filósofo francés Yves Zarka (2016), denomina como la agenda de reformulación ante realidades que emergieron como producto de la violencia occidental moderna, o en contra de dicha violencia, que, dicho sea de paso, parece haber sido leída, sobre todo en los círculos del pensamiento político liberal, como un simple efecto colateral de las bondades del proyecto moderno y de su gran aliado: el sistema económico-político capi- necessary, desirable, and that, seen with eyes of hope, it could pave the way toward a new conception of law, closer to the realities of the Colombian Caribbean and, therefore, to the physical and symbolic geographies of our south.

Keywords: Economic Inequality; Racial Inequality; Right to Development; Socioeconomic Rights; Economic Violence; and Justice.

talista. Hoy sabemos que las pretendidas bondades no lo eran en un sentido absoluto, y que los efectos colaterales de la Modernidad fueron más bien asuntos nucleares para algunas culturas que nunca fueron, ni son, Modernas, para parafrasear al filósofo Bruno Latour (2012).

Desde luego, de todo lo anterior han tomado atenta nota las autoras y los autores de Reduciendo desigualdades. Otros derechos y otros desarrollos que, bajo la edición académica de Nina Ferrer Araujo, y bajo el sello de la Universidad de San Buenaventura, fue publicado en 2020. La reseña en torno a esta contribución académica, busca responder a tres preguntas, a saber: ¿Cuál es la transición científica de la que se ocupa este libro? ¿Cuál es el orden de exposición que propone? Y derivado de lo anterior, ¿cuál es la visión de fondo que nutre a la obra en general y que por ende se refleja en cada capítulo?

La clave de la respuesta a la primera pregunta está en el subtítulo. Esta es, pues, una contribución dirigida en principio a un público interesado en reformular dos cosas a la vez: a) al derecho como disciplina, concepto y práctica y b) al desarrollo como campo de estudio y práctica discursiva con poderosos efectos en la realidad. Pero, sobre todo, es un libro que convoca a las personas preocupadas por y ocupadas de hacer inteligibles, comunicables y claros los cruces entre derecho y desarrollo, o derecho al desarrollo en perspectiva crítica y alternativa. No es como tal un libro de sociología, filosofía o antropología del derecho y del desarrollo, sino una apuesta de convergencia entre estas tres miradas y otras muchas que, no obstante, toma como materia prima a la realidad misma con sus cifras oficiales, con sus 
narrativas silenciadas, con sus genocidios estadísticos, sus cuestiones disputadas y sus laboratorios para componer otros mundos menos desiguales y menos oprimidos.

En cuanto a la segunda pregunta, el orden de exposición que la publicación sugiere está dado por juegos de duplas. Los dos primeros capítulos (la primera dupla), "Pobreza y desigualdad: la necesidad de una teoría crítica de la justicia global" escrito por el abogado y filósofo Juan Sebastián Carcía-Acevedo, y "Mujeres resistentes e insurgentes: alternativas contrapedagógicas contra la violencia económica" escrito por la editora y derivado de su investigación doctoral en humanidades; son una invitación a recorrer autores clásicos y trabajos recientes en torno a lo que se deja entrever en los títulos, pero sin perder de vista ni un solo instante las realidades empíricas con las que ponen a dialogar los marcos teóricos escogidos. En el primero, García-Acevedo va a la búsqueda de otra vía de la justicia global, desmarcada de la postura liberal de John Rawls y, para ello, traza un hilo de continuidad entre algunas formulaciones completamente vigentes de Karl Marx, la Teoría Crítica y los trabajos del estudiante rebelde de Rawls, es decir, los trabajos del profesor de Yale, Thomas Pogge (Hamburgo, 1953). Este primer capítulo es, entonces, una síntesis de lo mejor de los enfoques críticos en clave de una justicia global pensada y actuada por fuera del marco liberal. En el segundo, Ferrer Araujo nos confiesa cuál es el itinerario existencial (personal y académico) que ha atravesado su interés de investigar acerca de la violencia económica contra las mujeres en general, y contra las mujeres del Caribe colombiano, especialmente de Cartagena de Indias, en particular. Como en el caso de Carcía-Acevedo, en este segundo capítulo Ferrer Araujo recoge y comunica generosamente una síntesis de las principales posturas teóricas que en América Latina se han ocupado de develar el sutil nexo entre Estado, Capitalismo y Patriarcalismo y, sin reparos, de la mano de los trabajos de Rita Segato (Buenos Aires, 1951) y de otras ineludibles académicas y activistas que no se mencionarán aquí para dejar abierta una cierta actitud de curiosidad, da un paso más para hablar en tono afirmativo de la insurgencia de las mujeres como una pedagogía de la vida y de los vínculos, capaz de hacer frente y derrocar la tiránica pedagogía de la crueldad de un sistema capitalista que todo lo que toca lo vuelve cosa y mercancía, incluso la vida misma, y cuyas principales víctimas han sido las mujeres: Oprimidas pero no vencidas, como diría Silvia Rivera Cusicanqui (1986). Este capítulo es la bitácora de una investigadora doctoral y una fina provocación epistemológica.

La segunda dupla está constituida por historias de luchas sociales y políticas que subyacen a avances normativos de dos grupos de colectivos humanos: los pueblos indígenas y las comunidades LGBTIQ. En "Ciudadanía diferenciada, pluralismo, multiculturalismo y derecho de los pueblos indígenas en Colombia" (capítulo III del libro), el abogado, especialista en Derecho Constitucional y Maestro en Derecho Guillermo Araque Bermúdez pone en tensión la noción liberal de "persona jurídica" inherente a las interpretaciones jurisprudenciales más usuales a la luz del reclamo cultural que se hace en torno a un fundamento jurídico más colectivo, a saber: "pueblo indígena”. En el fondo, los pueblos indígenas resisten a una mirada del derecho que se basa en la idea de un individuo autónomo propia del liberalismo radical que, sin embargo, sigue siendo hegemónica. El capítulo ofrece, además, una síntesis de los principales avances normativos alcanzados por la vía de la lucha política de los pueblos indígenas en Colombia. Así mismo, en "Derechos humanos y ciudadanía LGBTIQ en Colombia” (capítulo IV del libro), Nina Ferrer y la internacionalista Diandra Payares forman un tándem para dibujar los avances internacionales y nacionales en materia de protección y defensa de los derechos de lesbianas, gais, bisexuales, transexuales e intersexuales tanto a escala internacional como nacional. Este capítulo es una síntesis histórica, teórica y normativa del movimiento LGBTIQ, es decir, una historia social del derecho otro.

Finalmente, la tercera dupla la conforman dos capítulos que buscan ejemplificar algunas implicaciones prácticas del cruce entre otros derechos y otros desarrollos. En "La interseccionalidad como herramienta para el diseño de políticas públicas de igualdad de género" (capítuloz del libro), el equipo de abogados conformado por Laura Romero López, 
Daniel Villegas Támara y Ramón Medina Arteta se encargan de exponer sus análisis acerca de la Política de Equidad de Cénero en el Departamento de Bolívar (Colombia) a la luz del principal aporte del feminismo negro, es decir, el enfoque interseccional (raza, clase, género, rol, entre otros). ¿Qué significa en realidad ser una persona interseccionada, a la vez mujer, negra y pobre? ¿Cómo lee la política pública estas intersecciones? Este capítulo ofrece poderosas pistas para el diseño de políticas públicas de la mano de las mujeres negras, afrocolombianas, raizales y palenqueras en Colombia. La autora y los autores hacen denuncias sobre los sistemas de información estadística sobre población negra y, por tanto, contribuyen a brindar criterios de consideración estadístico-política para la toma de decisiones públicas realmente interesadas en mejorar la situación actual de la mujer negra que habita y cohabita en el país. Por último, en "Laboratorios de innovación ciudadana, nuevas formas de cooperar: pinceladas en Cartagena de Indias" (capítulozrdel libro) las maestras en Cooperación Internacional para el DesarroIlo, Leivis Pua De La Hoz e Ibelis Blanco-Rangel, nos sumergen en el amplio territorio de los Living Labs y, con ello, como lo señalan en la introducción del trabajo: "Este capítulo pretende introducir al lector en el surgimiento de los laboratorios de innovación ciudadana (LI), su vinculación con el desarrollo sostenible y la manera en que estos se perfilan en escenarios de la participación ciudadana para la resolución de problemas sociales individuales o colectivos. La Cooperación Internacional para el Desarrollo (CID) ha intervenido en la resolución de estos problemas cuando las políticas públicas no han sido efectivas" (p. 147). Las autoras presentan y desarrollan casos que han sido de interés en Cartagena de Indias, pero, ante todo, este capítulo, como lo señala el epígrafe con el que inicia y se espera que algún día pueda ser consultado por el público lector, busca exponer que allí donde fracasan las políticas públicas, mientras exista un grupo humano comprometido con el grupo mismo, sin importar su escala, será posible activar la solidaridad internacional entre los pueblos.

Para responder a la tercera pregunta que se hacía y que, se interroga acerca de la visión de fondo que nutre al libro Reduciendo desigualdades. Otros derechos y otros desarrollos, puede decirse que: debido al diálogo fecundo entre autores, ideas y planteamientos clásicos con autores, ideas y planteamientos recientes en cada una de las partes, temas y problemas que conforman esta publicación, y cuyo común denominador es el desmonte del marco liberal del derechoy del desarrollo que depositó toda su confianza y toda su razón de ser en la promoción del individualismo económico-jurídico y en el crecimiento económico de esos egos aislados, este libro abre camino para devolvernos, en palabras del premio nobel de literatura de 1913, del indio Rabindranath Tagore, "el derecho de entrar en la gran fiesta de la común [no individualista] vida humana" (Tagore, 1959: p. 9). Lo recomiendo ampliamente para la persona dispuesta a ir más allá de la forma dada, es decir, para la persona interesada en trans-formar la injusta realidad del capitalismo actual.

\section{Referencias}

Latour, B.(2012). Nunca fuimos modernos. Ensayos de antropología simétrica. Buenos Aires: Siglo XXIeditores.

Rivera Cusicanqui, S. (1986). Oprimidos pero no vencidos: Luchas del campesinado aymara y qhechwa de Bolivia, 1900-1980. Ceneva: Instituto de Investigaciones de las Naciones Unidas para el Desarrollo Social.

Tagore, R. (1959). Gitanjali, La Luna Nueva y El Jardinero. España: Ediciones Zeus.

Zarka, Y. (2016). La inapropiabilidad de la tierra: Principio de una refundación filosófica frente a los desafios de nuestro tiempo. Barcelona: Nuevos Emprendimientos Editoriales (NED). 